ARTICLE

https://doi.org/10.1038/s41467-019-12863-6

OPEN

\title{
Photo-accelerated fast charging of lithium-ion batteries
}

Anna Lee ${ }^{1}$, Márton Vörös², Wesley M. Dose, Jens Niklas (1) ${ }^{1}$, Oleg Poluektov (1) ${ }^{1}$, Richard D. Schaller (10 ${ }^{3}$, Hakim Iddir ${ }^{1}$, Victor A. Maroni ${ }^{1}$, Eungje Lee (i) ${ }^{1}$, Brian Ingram (1) ${ }^{1}$, Larry A. Curtiss ${ }^{2} \&$ Christopher S. Johnson (i) ${ }^{1 \star}$

Due to their exceptional high energy density, lithium-ion batteries are of central importance in many modern electrical devices. A serious limitation, however, is the slow charging rate used to obtain the full capacity. Thus far, there have been no ways to increase the charging rate without losses in energy density and electrochemical performance. Here we show that the charging rate of a cathode can be dramatically increased via interaction with white light. We find that a direct exposure of light to an operating $\mathrm{LiMn}_{2} \mathrm{O}_{4}$ cathode during charging leads to a remarkable lowering of the battery charging time by a factor of two or more. This enhancement is enabled by the induction of a microsecond long-lived charge separated state, consisting of $\mathrm{Mn}^{4+}$ (hole) plus electron. This results in more oxidized metal centers and ejected lithium ions are created under light and with voltage bias. We anticipate that this discovery could pave the way to the development of new fast recharging battery technologies.

\footnotetext{
${ }^{1}$ Chemical Sciences and Engineering Division, Argonne National Laboratory, Lemont, IL 60439, USA. ${ }^{2}$ Material Sciences Division, Argonne National Laboratory, Lemont, IL 60439, USA. ${ }^{3}$ Center for Nanoscale Materials, Nanoscience and Technology Division, Argonne National Laboratory, Lemont, IL 60439, USA. *email: cjohnson@anl.gov
} 
ithium-ion batteries (LIBs) must be slow-charged in order to restore the full capacity (stored energy) of the battery, as well as to promote longer battery cycle life. Depending on the physicochemical properties of the composite electrodes, fast DC charging is in principle feasible when engineered active particle physical morphologies ${ }^{1,2}$, or low electrode loadings of nanostructured materials are used ${ }^{3,4}$. However, these approaches result in limited volumetric energy densities (or the amount of stored energy per volume of material) within the battery pack. A new paradigm in battery technology is required in order to overcome these obstacles leading to improved battery lifetime and requisite fast charging, particularly for battery-only electric vehicles and portable electronics.

Lithium-transition metal oxide materials such as $\mathrm{LiMn}_{2} \mathrm{O}_{4}$ (LMO) are widely employed as cathodes for energy-storage applications ${ }^{5-9}$. One such material is $\mathrm{LiMn}_{2} \mathrm{O}_{4}$ oxide (henceforth abbreviated as LMO) with the spinel structure ${ }^{10}$. LMO is a technologically relevant cathode for LIBs due to only $6 \%$ volume change during lithium intercalation reaction ${ }^{10}$. The removal of one lithium atom per formula unit ( $x=1$ limit) yields the cubic $\lambda-\mathrm{Mn}_{2} \mathrm{O}_{4}$ phase ${ }^{10-12}$, as shown in Eq. 1).

$$
\mathrm{LiMn}_{2} \mathrm{O}_{4} \rightarrow \mathrm{Li}_{(1-x)} \mathrm{Mn}_{2} \mathrm{O}_{4}+\mathrm{xLi}^{+}+\mathrm{xe}^{-}(0<x<1)
$$

During cycling both lithium cations and electrons must flow into and out of the material. For charging (moving to the rhs of Eq. 1), LMO is bulk oxidized, lithium leaves the material, and lithium cations are reduced with lithium metal deposition at the opposite anode (in this case lithium metal); the electrons travel in the external circuit with high average potential $\sim 4.1 \mathrm{~V} v s . \mathrm{Li}^{+/ o}$. Mn oxidation state of LMO will change from $\mathrm{Mn}^{3+}$ to $\mathrm{Mn}^{4+}$ depending on the state of charge of the battery and the amount of $\mathrm{Li}$ in the material ${ }^{10,13}$. Stoichiometric LMO is a mixed valence compound with an average $\mathrm{Mn}$ oxidation state of +3.5 ; the electronic conductivity is mediated through polarons, and the bandgap is about $2-3 \mathrm{eV}$ (Supplementary Fig. 1) ${ }^{14,15}$. As such, broadband white light (henceforth abbreviated light) should create electron-hole polaron pairs in LMO, which then could charge separate under potential bias.

We report here that illumination of a spinel-type $\mathrm{LiMn}_{2} \mathrm{O}_{4}$ cathode induces efficient charge-separation leading to fast lithium-ion battery charging. The discovery that exposure of LMO to light lowers charge transport resistance can lead to new fast recharging battery technologies for consumer applications and battery-only electric vehicles.

\section{Results}

Light-accepting battery design. To probe the process, an 'open' light-accepting coin cell battery was developed. A schematic of our 'open' cell is illustrated in Fig. 1a wherein a transparent quartz window allows light to enter the cell. Figure $1 \mathrm{~b}$ represents a magnified view of the LMO electrode with active particles, binder, and carbon conductive matrix. Galvanostatic cycling measurements confirm the operation of the 'open' cell (Fig. 1c), which is consistent with a conventional cell (Supplementary Fig. 2). The voltage profiles are as expected, whereby the reaction occurs over a voltage window of 3.2 to $4.4 \mathrm{~V} v s$. $\mathrm{Li}^{+/ \mathrm{o}}$. The amount of capacity obtained is about $90 \%$ of the theoretical maximum, which is consistent with the literature ${ }^{10,12,16}$.

Electrochemical performance. In the electrochemical experiment, the 'open' cell is kept in either a 'light-off state or a 'light-on' state, which is irradiated by white light from a Xe lamp fitted with an IR filter (see Methods). A direct-current (DC) experiment is carried out whereby a constant voltage of $4.07 \mathrm{~V}$ is applied to the lightoff state cell (blue curve in Fig. 2a), and the current is measured as a function of time. The applied voltage of $4.07 \mathrm{~V}$ (about $50 \%$ stateof-charge SOC) was chosen so as to provide a reasonable capacity from the battery cell, while also delivering a modest current over experimental time. As expected, a decay of current in time occurs as the reaction proceeds, removing $\mathrm{Li}$ cations from LMO and oxidizing $\mathrm{Mn}$ redox centers. The amount of charge from the cell is $2.21 \mathrm{C}$ (or $26.1 \mathrm{mAhg}^{-1}$ based on the oxide active weight $=23.5 \mathrm{mg}$ ). From the galvanostatic result in Fig. 1c, the amount of capacity expected at $4.07 \mathrm{~V}$ should be $\sim 65 \mathrm{mAh} \mathrm{g}^{-1}$; clearly the light-off state cell only reaches $\sim 42 \%$ of this value. Indeed, even after $22 \mathrm{~min}$ the current is still $1.2 \mathrm{~mA}$ and the reaction continues. In contrast, integrating the charge passed over $22 \mathrm{~min}$ gives a capacity of $4.36 \mathrm{C}\left(\sim 52 \mathrm{mAh} \mathrm{g}^{-1}\right)$ for the 'light-on' state (red curve in Fig. $2 \mathrm{a}$ ), $\sim 92 \%$ of the practical capacity.

The cell exhibits normal function after the light test and further, Raman spectroscopy of the LMO material suggests no phase changes, nor electrolyte decomposition/change (Supplementary Fig. 3). The UV/Vis absorption spectrum of the electrolyte was measured and was found to be transparent over the visible region of the spectrum, with minimal absorption in the UV portion. The spectral output of the Xe lamp used in the work has $<5 \%$ overall energy emitted in the UV region. With little light absorption across this spectral region, it is accurate to predict no overt degradation of the electrolyte under long term light exposure. a

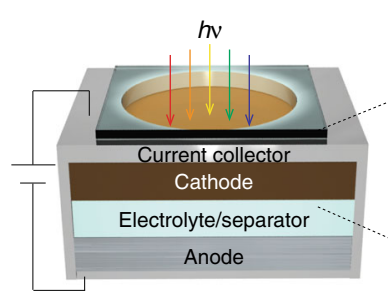

b

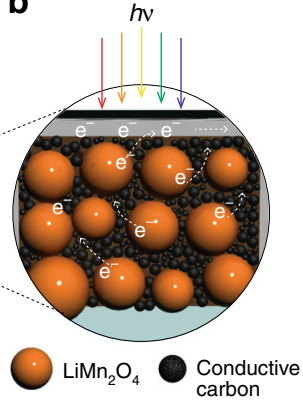

C

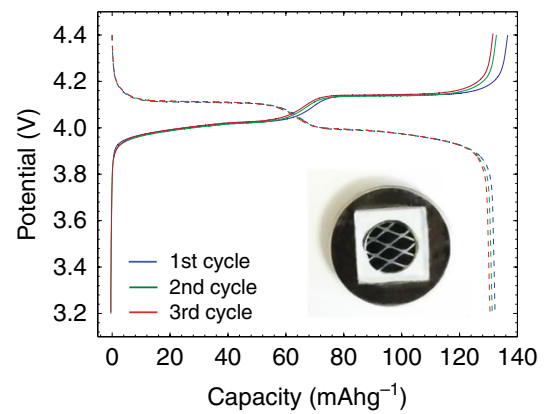

Fig. 1 The principle of a photo-accelerated lithium-ion battery cell. The cell consists of a transparent window, current collector, cathode, electrolyte, separator, and anode. The broadband white light is used with an IR filter to avoid undesired heating of the cell. b Magnified view of the composite LMO cathode consisting of a Teflon binder, (e.g. $\left.-\left(\mathrm{CF}_{2}\right)_{\mathrm{n}}-\right)$, carbon particles as conductive diluent (e.g. acetylene carbon black), and active oxide powder. c Galvanostatic cycling voltage profile of a Li ||1.2 M LiPF $;$ EC:EMC 3:7 (w:w) | LMO (EC=ethylene carbonate; EMC= ethyl methyl carbonate). Three cycles of charging (indicated in solid lines) and discharging (in dash lines) profiles between 3.2 and $4.4 \mathrm{~V}$ at a $\mathrm{C} / 10$ rate are shown. $\mathrm{A}$ photograph of a fabricated 'open' cell is shown in the inset 

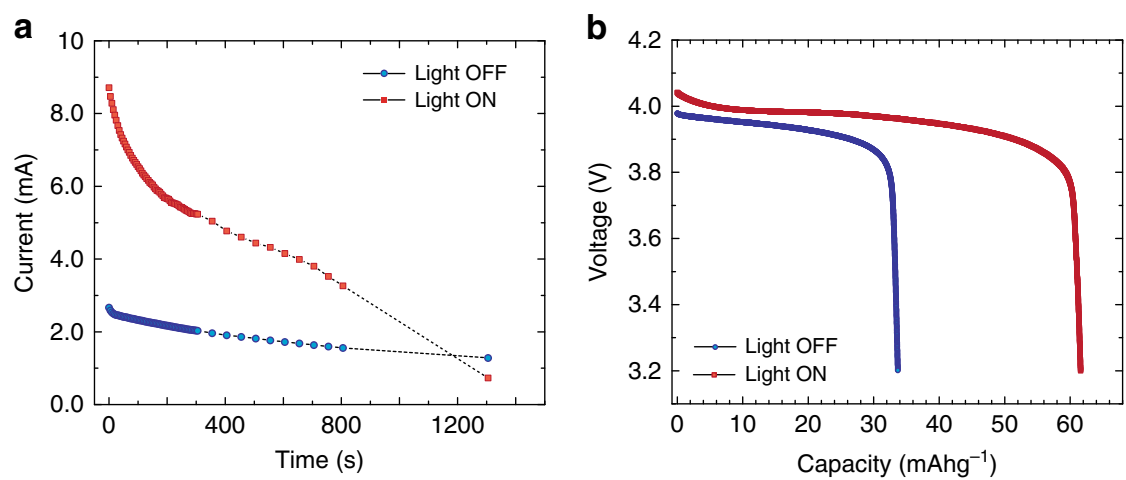

Fig. 2 Electrochemical performance of the light accepting 'open' lithium-ion battery cell. Li || 1.2 M LiPF $;$ EC:EMC 3:7 (w:W) | LMO (EC=ethylene carbonate; $E M C$ = ethyl methyl carbonate). a Chronoamperometry curves for 'light-on' vs. 'light-off' state during constant voltage hold charging at $4.07 \mathrm{~V}$ (vs. $\mathrm{Li}^{+/ 0}$ ) for approximately 22 minutes. b Constant discharge curves carried out without illumination after the chronoamperometry experiment shown in a (voltage profiles at $\mathrm{C} / 10)$. The "light-on" experiments were carried out under $1 \mathrm{SUN}\left(100 \mathrm{~mW} / \mathrm{cm}^{2}\right)$, a white light is used with an IR filter to avoid undesired heating of the cell. The heat effect in control experiments are presented in Supplementary Fig. 10

Galvanostatic (constant current) discharge without illumination (Fig. 2b) after the voltage hold experiments confirms that $\mathrm{Li}$ cations were de-intercalated from the LMO and the discharge capacity during $\mathrm{Li}$ re-insertion is consistent with the chronoamperometric (constant voltage charge) response in Fig. 2a. For this particular device, approximately twice the battery capacity is observed for the 'light-on' vs. 'light-off condition. This corresponds to an increase of the charging rate by a factor of 3.4 compared to the light-off state. Light enhancement has been reproduced in multiple devices with charging rate enhancement magnitudes ranging from 1.7 to 3.4 (Supplementary Fig. 4).

The current response delivered from 0 to $100 \%$ SOC (state-ofcharge) was analyzed in potential step experiments in a sealed cell (Supplementary Fig. 5). Briefly the charge rate is near linearly flat over different SOC amounts up to $91 \%$ SOC. At the end of charge, about 10-100\% higher currents are achieved between 91 and $99 \%$ SOC. At $99 \%$ SOC the charge rate decreases precipitously to below $10 \%$ of that from 0 to $44 \%$ SOC. This indicates that $\mathrm{LMO}$ is not limited in intrinsic charging rates at end of charge. Thus the light-induced photo-current provides the extra route to faster charging time in addition to the observed current driven potentiostatically.

Transient absorption spectroscopy, electron paramagnetic resonance, and density functional theory calculations. To probe mechanistic aspects of the light induced electrochemical processes in $\mathrm{LiMn}_{2} \mathrm{O}_{4}$, transient absorption (TA) spectroscopy, electron paramagnetic resonance (EPR), and density functional theory (DFT) calculations were investigated. The transient absorption map, spectra, and dynamics of a sputtered LMO thin-film only without other components in the composites (Supplementary Fig. 6) using $3.2 \mathrm{eV}(390 \mathrm{~nm})$ pump photons and a white light probe in a transmission geometry are shown in Fig. 3a-c, respectively. Optical excitation clearly results in a broad photoinduced absorption signal with a maximum near $2.8 \mathrm{eV}(442 \mathrm{~nm})$ extending down to $2.1 \mathrm{eV}(590 \mathrm{~nm})$. The induced absorption signal is consistent with generation of electron-hole pairs in the LMO film that can yield the modified charging rates in the operating illuminated cell. Importantly, the TA signal has longlived components, with a trace that is characterized by decay components $\sim 0.066,2.5$, and $10.3 \mu \mathrm{s}$. Indeed, fully operational LMO cathode composites show similar dynamic timescales in the transient diffuse reflectance (Fig. $3 \mathrm{c}$ inset). Similar to photovoltaic devices that require long lived excited states ${ }^{17}$, such long-lifetime may be beneficial for light-induced modification of a battery system.
To determine the chemical changes induced by optical excitation, EPR measurements were carried out with and without illumination at $10 \mathrm{~K}$ on a LMO electrode that had been potentiostatically charged at $4.07 \mathrm{~V}$, then extracted from the cell. Without illumination ('light-off state), a broad signal with a line width of several hundred $\mathrm{mT}$ was observed, centered at $\mathrm{g} \approx 2$ $(\approx 0.34 \mathrm{~T})$ corresponding to paramagnetic $\mathrm{Mn}^{4+}$ ions interacting with many close-by paramagnetic ions, like other $\mathrm{Mn}^{4+}$ or $\mathrm{Mn}^{3+}$ ions (Fig. $3 \mathrm{~d})^{18,19}$. Note that trivalent $\mathrm{Mn}^{3+}$ ions themselves are not directly observable under the experimental conditions. For the 'light-off state, a narrow and intense signal centered at $340 \mathrm{mT}$ (corresponding to $g \approx 2$ ) is observed that we attribute to a radical species in the carbon/binder part of the composite cathode. The six-line signal with $\sim 50 \mathrm{mT}$ total width corresponds to $\mathrm{Mn}^{2+}$ ions ${ }^{20-23}$. The build-up of a separate $\mathrm{Mn}^{2+}$ phase has been reported for $\mathrm{Li}$ extraction from $\mathrm{LiMn}_{2} \mathrm{O}_{4}$ spinel ${ }^{24}$. Since $\mathrm{Mn}^{2+}$, even in small concentration, gives intense signals, the actual amount of $\mathrm{Mn}^{2+}$ impurities in the battery material may be relatively small. According to the spin quantification of the EPR spectra, the relative concentration of the $\mathrm{Mn}^{2+}$ ions is at least 3 orders of magnitude less than concentration of $\mathrm{Mn}^{4+}$ ions. Upon illumination ('light-on' state), a new distinctive broad signal with a width of $\sim 215 \mathrm{mT}$ (narrower than the broad 'light-off $\mathrm{Mn}^{4+}$ signal) is created. This type of signal is typical for $\mathrm{Mn}^{4+}$ in $\mathrm{LMO}$, but has different coordination and/or magnetic surrounding than the 'light-off $\mathrm{Mn}^{4+}$ signal ${ }^{18,19}$. Note, that the intensity of the $\mathrm{Mn}^{2+}$ signal does not change upon illumination. Figure $3 \mathrm{e}$ shows that the signal corresponding to excess $\mathrm{Mn}^{4+}$ is created rapidly after the illumination starts, then it plateaus, and finally it drops close to its 'light-off state after illumination is turned off. More importantly, the growth in the number of $\mathrm{Mn}^{4+}$ ions upon illumination indicates that $\mathrm{Mn}^{3+}$ is oxidized to $\mathrm{Mn}^{4+}$ (see Methods and Supplementary Figs. 7 and 8 on the effect of a control system).

\section{Discussion}

From our experimental results, we propose a mechanism by which light assists fast charging of LMO. Upon illumination, photoexcited $\mathrm{Mn}^{3+}\left(\left[\mathrm{Mn}^{3+}\right]^{\star}\right)$ leads to the formation of $\mathrm{Mn}^{4+}$ (hole) and electron (Eq. 2). Specifically, under potential bias the electron percolates through the structure via charge transfer/polaron hopping towards the current collector, where the electron passes into the external circuit. This process is facilitated by the presence of the conductive carbon electrode network. In essence, from the photochemical process more $\mathrm{Mn}^{3+}$ is being oxidized to $\mathrm{Mn}^{4+}$ and subsequently more $\mathrm{Li}^{+}$ions are ejected 
a

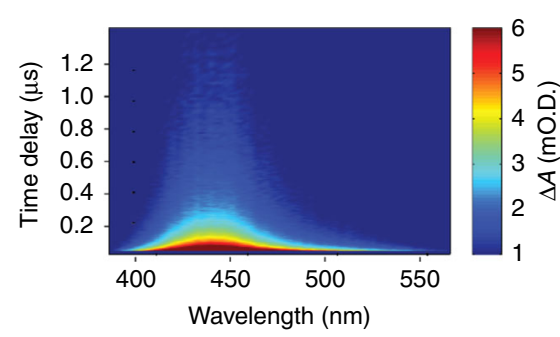

b

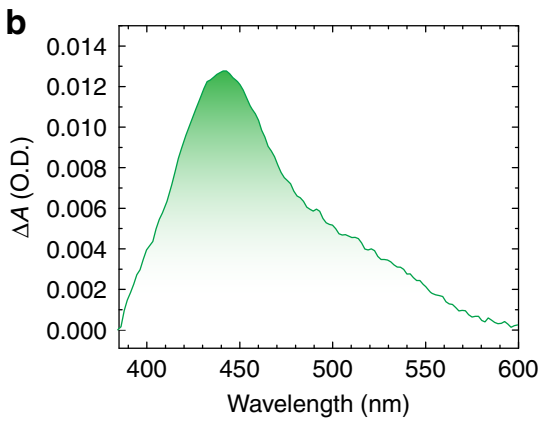

C

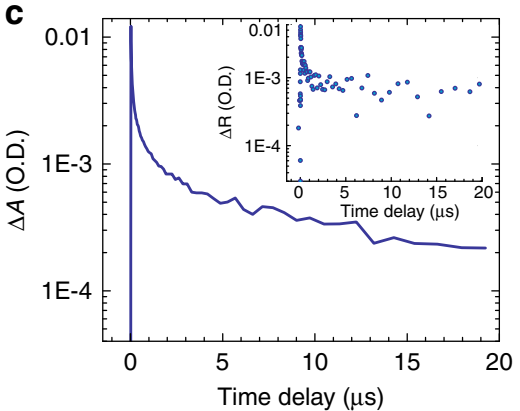

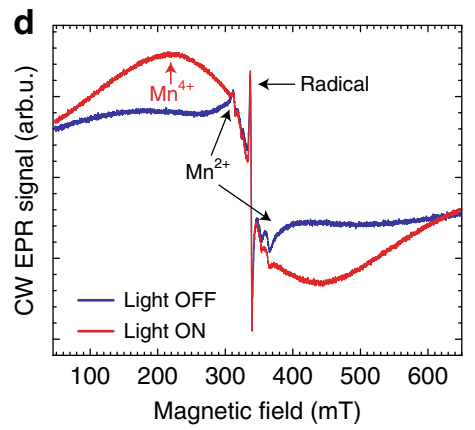

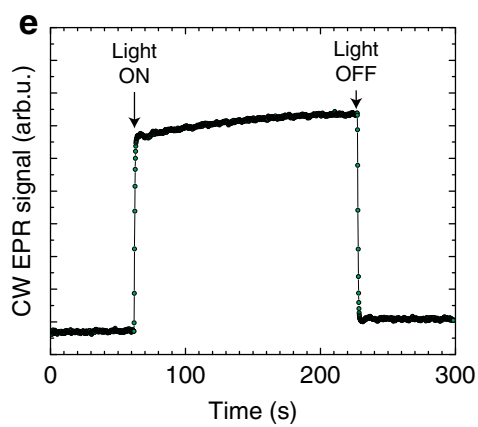

Fig. 3 Probing the light induced processes through transient absorption (TA) and electron paramagnetic resonance (EPR) measurements. a A TA map, b spectrum at $0.026 \mu$ s pump-probe time delay, and c dynamics at $448 \mathrm{~nm}$ for an LMO film using $3.2 \mathrm{eV}$ ( $390 \mathrm{~nm}$ ) pump photons. Inset shows transient diffuse scattering experiments performed on a fully operational LMO composite cathode. $\mathbf{d}$ Continuous wave (CW) X-band EPR spectra of a charged LMO battery cathode in the 'light-off' state (i.e., before illumination shown in blue spectrum) and 'light-on' state (i.e., during illumination in red spectrum) with white light. $T=10 \mathrm{~K}$. The CW EPR results in a derivative-type lineshape. e, Time dependence of the EPR signal at $210 \mathrm{mT}$ before, during ('light-on'), and after illumination ('light-off')

from the structure (i.e. the faster charging) than in the dark state. EPR indeed shows that, under photochemical conditions, the amount of oxidized $\mathrm{Mn}^{4+}$ species is found to be greater with "light-on" than "light-off" (Fig. 3d, e).

$$
\begin{gathered}
\mathrm{Mn}^{3+} \stackrel{\mathrm{h} v}{\longrightarrow}\left[\mathrm{Mn}^{3+}\right]^{*} \approx \mathrm{Mn}^{4+}(\text { hole })+e^{-}(\text {electron }) \\
2 \mathrm{Mn}^{3+} \stackrel{\mathrm{h} v}{\longrightarrow} 2\left[\mathrm{Mn}^{3+}\right]^{*} \approx \mathrm{Mn}^{4+}+\mathrm{Mn}^{2+}
\end{gathered}
$$

In a parallel process, disproportionation reaction (Eq. 3) could also be a possibility occurring at the particle surface forming $\mathrm{Mn}^{4+}$ and $\mathrm{Mn}^{2+}$. Disproportionation reactions are calculated to occur at a surface of LMO enriching $\mathrm{Mn}^{3+}$ sites at an electrochemical surface environment ${ }^{25}$. Since light irradiation is shown (EPR) to produce $\mathrm{Mn}^{4+}\left(\mathrm{Mn}^{3+}\right.$ photo-oxidation), on static $\mathrm{Mn}^{3+}$ sites, without any further effect on the distribution and ordering of the rest of the Mn ions in the material. This process will in turn increase the local (surface) concentration of $\mathrm{Mn}^{4+}$ and reduce $\mathrm{Mn}^{3+}$, thereby reducing even further the possibility of $\mathrm{Mn}^{3+}$ disproportionation reaction that inherently requires a higher concentration of $\mathrm{Mn}^{3+}$. In fact DFT calculations show that disproportionation of $\mathrm{Mn}^{3+}$ on the surface would occur only for very specific configurations (local distribution of $\mathrm{Mn}^{3+}$ and $\left.\mathrm{Mn}^{4+}\right)$. Hence, we do not expect EPR to detect any $\mathrm{Mn}^{2+}$ in the static mode (no charging), or in the right-hand side of Eq. 3.

Instead, under voltage hold bias, the current flows via a polaron conduction process, where $\mathrm{Mn}^{4+}$ and $\mathrm{Mn}^{3+}$ continuously exchange charge, and creating new arrangements and ordering of $\left(\mathrm{Mn}^{3+}-\mathrm{Mn}^{4+}\right)$ patterns, increasing the electronic entropy of the material. The shuffling between the $\mathrm{Mn}^{4+}$ and $\mathrm{Mn}^{3+}$ allows for a continuous and an increase sampling of new configurations that will be favorable for $\mathrm{Mn}^{3+}$ disproportionation reaction. The energy provided by light absorption will further help the reaction to occur (last step in green shaded region in Fig. 4c DFT energy profile). Note that the broad band of light will provide absorption even at lower wavelengths than required for photo-oxidation. Absorption at low energies can be enough to help disproportionation, which further increases the carrier density.

Note that delithiation (charge) is a process in which an electron and $\mathrm{a} \mathrm{Li}^{+}$are removed from the material at the same time. DFT results show that the energy required to remove a $\mathrm{Li}^{+}$from the material is reduced near higher concentrations of $\mathrm{Mn}^{4+}$ (allowing for fast charging), but also even more reduced near $\mathrm{Mn}^{2+}$ (product of $\mathrm{Mn}^{3+}$ disproportionation). This later result, suggest a very short lifetime for $\mathrm{Mn}^{2+}$ during charging.

Overall, both photo-oxidation and disproportionation alone allow for fast charging, however, the combination of both processes is likely to be more efficient, as the applied bias will continuously feed and replenish the surface with fresh $\mathrm{Mn}^{3+}$ sites that will undergo photo-oxidation and disproportionation.

To support the disproportionation part of the mechanism, we performed density functional theory (DFT) calculations. Figure 4 shows the structural model of bulk LMO with and without disproportionation and the transition path between the ground state and disproportionated state (The details for model optimizations are in Supplementary information and Methods). The ground state structure of bulk LMO contains alternating and ordered $\mathrm{Mn}^{3+}$ and $\mathrm{Mn}^{4+}$ sites $^{15,26}$. In order for disproportionation to happen this oxidation state pattern needs to be disordered by electron transfer processes as shown in the first steps of the transition path. The disproportionated structure is $\sim 0.45 \mathrm{eV}$ higher in energy than the ground state. The overall barrier energy of disproportionation is only about $0.5 \mathrm{eV}$, at least $1.5 \mathrm{eV}$ lower than the optical gap of the system $(\sim 2 \mathrm{eV})$. This suggests that the light can provide the necessary excess energy to climb the calculated barrier. We find that $\mathrm{Li}$ extraction from the $\mathrm{Mn}^{3+} / \mathrm{Mn}^{4+}$ disordered or disproportionated LMO is energetically more favorable than extracting $\mathrm{Li}$ from $\mathrm{Mn}^{3+} / \mathrm{Mn}^{4+}$ ordered LMO 
a

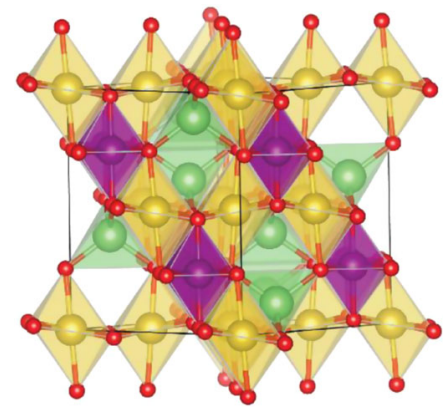

C

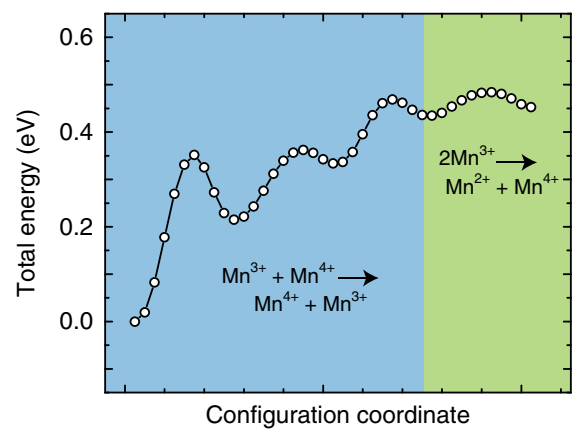

b

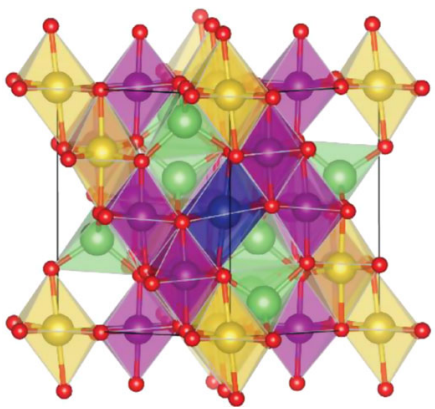

d

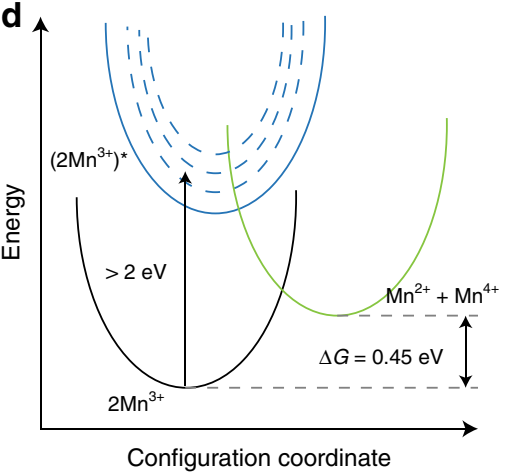

Fig. 4 Atomistic studies of the proposed photo-induced charge separation mechanism in LMO. $\mathbf{a}, \mathbf{b}$ Structural model of bulk stoichiometric LMO without and with disproportionation, respectively. Green, red, purple, yellow, blue spheres represent $\mathrm{Li}, \mathrm{O}, \mathrm{Mn}^{4+}, \mathrm{Mn}^{3+}$, and $\mathrm{Mn}^{2+}$ sites, respectively. c Relative ground state total energy along the transition path from the ground state to the disproportionated state. The first transition is a concerted electron exchange between two $\mathrm{Mn}^{3+}$ and $\mathrm{Mn}^{4+}$ sites, the second and third transition are single electron exchange processes between $\mathrm{Mn}^{3+}$ and $\mathrm{Mn}^{4+}$, and the last transition is the disproportionation reaction. $\mathbf{d}$ Schematic of the Eq. 3 scenario where photo-excited electron transfer process occurs from the ground state, through the excited states $\left(2\left[\mathrm{Mn}^{3+}\right]^{\star}\right)$ to the disproportionated state $\left(2 \mathrm{Mn}^{3+} \rightarrow \mathrm{Mn}^{2+}+\mathrm{Mn}^{4+}\right)$

without disproportionation (Supplementary Fig. 9). This might also have implications in build-up of $\mathrm{Mn}^{3+}$ at the electrode surface that can undergo disproportionation and follow-up electrochemical reaction more easily in the presence of light.

In summary, we have shown that light irradiation dramatically increases the charging rates of LMO in a conventional lithiumion battery configuration under a potential bias. This is different than previous studies ${ }^{27-29}$ that combine photovoltaic and battery materials into one device to produce photoelectric conversion and storage efficiency and incorporating light-active photoelectrode materials, redox mediators, or dyes into batteries. Our work introduces a completely new route for dynamically altering the active material characteristics in lithium-ion batteries, in this case through light - matter interactions. While we have observed a significant reduction in charge time for our system, the overall technology improvement to realize faster recharge rates will need to establish engineering controls on the overall battery pack including the optimization of anode and electrolyte. We believe that this photo-accelerated charging process will open pathways towards rapid charging in future battery technologies.

\section{Methods}

LIB design and preparation. Cathode electrodes were prepared from a mixture of a Teflon binder, (e.g. $\left.-\left(\mathrm{CF}_{2}\right)_{\mathrm{n}}-\right)$, carbon particles as conductive diluent (e.g., acetylene carbon black), and active $\mathrm{LiMn}_{2} \mathrm{O}_{4}$ (NEI, Grade BE-30) powder in a weight ratio of 20:5:75\%, respectively and the assemblage was optimized. The binder keeps particles in laminate form, the carbon provides a conductive network amongst the particles, and active material oxide acts to support the electrochemical reaction and act as lithium cation host. After replenishing with isopropanol, the mixture was well-mixed and ground with a mortar and pestle until it formed a selfstanding laminate. A thin sheet of laminate was achieved by manual rolling, after which it was dried at $75^{\circ} \mathrm{C}$ in an oven and stored under vacuum. The free standing electrode was obtained by placing the pre-cut laminate (14 $\mathrm{mm}$ in diameter) onto an $\mathrm{Al} \mathrm{mesh} \mathrm{(14} \mathrm{mm}$ in diameter), then the laminate and the mesh were pressed together under hydraulic pressure (Carver Laboratory Equipment). The top of a 2032 coin cell was modified by punching a hole $(8 \mathrm{~mm}$ in diameter) and then sealing the hole with Kapton tape prior to coin cell assembly. The modified coin cell was assembled with the cathode laminate, a glass fiber separator (Whatman ${ }^{\circledR}$ Glass microfiber filters, Grade GF/F), GenII electrolyte (1.2 $\mathrm{M} \mathrm{LiPF}_{6}$ in 3:7 ECEMC solution, Tomiyama Pure Chemical Industries), and a lithium metal anode (FMC Lithium) in an Argon-filled glove box. To probe an 'open' light-accepting coin cell battery, the tape was removed from the hole and a transparent quartz window (Technical glass products Inc.) was glued into place over the hole.

Light and electrochemical experiments set up. For the 'light' experiments, a $300 \mathrm{~W}$ Xenon lamp (Atlas Specialty Lighting with a Perkin Elmer power supply) that spans from $300 \mathrm{~nm}$ to $1100 \mathrm{~nm}$ was used as a white light source and an IR filter (Newport) was used to avoid undesired heating of the cell. The spectral output of the Xe lamp used in the work has $<5 \%$ overall energy emitted in the UV region. The incident light was normal to the sample and the temperature of the cell was measured by an IR thermometer focused on the transparent window. There was a temperature increase of $\sim 7^{\circ} \mathrm{C}$ when electrochemical measurements were carried out under $\sim 1$ SUN condition $\left(100 \mathrm{~mW} \mathrm{~cm}^{-2}\right)$. The heat effect in control experiments are presented in Supplementary Fig. 10. These results separate the heat contribution to the potentiostatic current due to thermal activation compared to photo-activation. Heating the 'open' cell at $35^{\circ} \mathrm{C}\left(10^{\circ} \mathrm{C}\right.$ higher $)$, increases the charge current by a factor 1.16 (not doubled), and at $45^{\circ} \mathrm{C}$, a factor of 1.26 was realized over that of $25^{\circ} \mathrm{C}$ in "light-off" condition.

Cycle performance of the modified cells and of conventional (un-modified) cells were compared by measurements involving galvanostatic charge and discharge of the cells between 3.2 and $4.4 \mathrm{~V}$ at various $\mathrm{C}$ rates ranging from $2 \mathrm{C}$ to $\mathrm{C} / 10$ (calculated based on a theoretical capacity of $148 \mathrm{mAh} \mathrm{g}^{-1}$ for $\mathrm{LiMn}_{2} \mathrm{O}_{4}$ ). The active $\mathrm{LiMn}_{2} \mathrm{O}_{4}$ material in an electrode typically weighed between 20 and $25 \mathrm{mg}$. Chronoamperometry was carried out by using a Gamry and Solartron SI1260 Frequency Response Analyzer. 'Light-on' and 'light-off state chronoamperometry measurements were performed by holding a constant voltage of $4.07 \mathrm{~V}$ (vs. a $\mathrm{Li}$ metal counter electrode). All relevant data processing/analysis was done in Matlab (Mathworks Inc., Natick).

Raman spectroscopy. Raman measurements were performed using a Renishaw inVia Raman microscope equipped with a $785 \mathrm{~nm}$ excitation laser. A $1200 \mathrm{l} / \mathrm{mm}$ grating was used in conjunction with a Ren 578 CCD detector. The laser power delivered to the sample surface was $<2 \mathrm{~mW}$; the laser spot diameter was 
approximately $10 \mu \mathrm{m}$. Spectra were recorded through the window of the cell $\left(\mathrm{BaF}_{2}\right.$ or quartz) using a 50-X objective with a numerical aperture of 0.5 . A total of ten coadded 20 second exposures were made at each of the probed spots in the rectangular map. Other details of the Raman measurements and the spectra processing/ analysis are the same as presented in refs. ${ }^{25,30-32}$

Absorption and transient absorption measurements. Absorption measurements were carried out using a Cary 5000 UV-Vis-NIR spectrophotometer. The UV/Vis absorption spectrum of the electrolyte was measured and was found to be transparent over the visible region of the spectrum, with minimal absorption in the UV portion. With little light absorption across this spectral region, it is accurate to predict no overt degradation of the electrolyte under long term light exposure.

Spectrally-resolved transient absorption (TA) measurements were performed using a Helios EOS spectrometer and an amplified Ti:sapphire laser operating at $1 \mathrm{kHz}$ that was tuned to produce $390 \mathrm{~nm}$ pump pulses using an optical parametric amplifier. White light probe pulses were derived from a picosecond Nd:YAG laser and photonic crystal fiber. A portion of the probe pulse was beamsplit to produce a reference laser shot for noise reduction. Pump-probe delay time was controlled and evaluated electronically. For TA measurements, two samples were used (a LMO only film as a control and LMO composite electrodes used in fully operational 'open' cells). For the LMO film preparation, a $200 \mathrm{~nm}$ film of LMO was deposited on a quartz substrate (Technical Glass Products, USA) with a commercial sputtering system (AJA International, USA). Deposition was conducted by RF magnetron sputtering of a stoichiometric LMO target ( $>90 \%$ density) in argon at $3.1 \mathrm{mTorr}$ and room temperature. The net RF power was $75 \mathrm{~W}$ and the deposition rate was $\sim 6 \AA \mathrm{min}^{-1}$. A lithium overpressure was provided by DC sputtering from a Li-metal target at $15 \mathrm{~W}$ set off axis by $20^{\circ}$. The deposition times were 9 hours for $\sim 300-350 \mathrm{~nm}$ films. The as-deposited LMO films were found to be amorphous; however a subsequent anneal air at $800{ }^{\circ} \mathrm{C}$ for $16 \mathrm{~h}$ resulted in a crystalline/ polycrystalline films.

Electron paramagnetic resonance (EPR) experiments. Continuous wave (CW) X-band (9-10 GHz) EPR experiments were carried out with a Bruker ELEXSYS II E500 EPR spectrometer (Bruker Biospin, Rheinstetten, Germany), equipped with a $\mathrm{TE}_{102}$ rectangular EPR resonator (Bruker ER 4102st). A helium gas-flow cryostat (ICE Oxford, UK) and an ITC503 from Oxford Instruments, UK, were used for measurements at cryogenic temperatures $(T=10 \mathrm{~K})$. Light excitation was done directly in the resonator with a $300 \mathrm{~W}$ Xenon lamp (LX $300 \mathrm{~F}$ from Atlas Specialty Lighting with PS300-13 $300 \mathrm{~W}$ power supply from Perkin Elmer). A water filter was used in combination with a KG2 short pass filter (Schott) to avoid unwanted heating of the sample. A GG400 long pass filter (Schott) was used to remove UV light. Data processing was done using Xepr (Bruker BioSpin, Rheinstetten) and Matlab 7.11.1 (The MathWorks, Inc., Natick) software. Simulations were performed using the EasySpin software package (version 5.0.20).

Density functional theory (DFT) calculations. We carried out our atomistic calculations using the plane-wave density functional theory (DFT) code QuantumEspresso $^{33}$. Electron-nuclei interaction was taken into account by using recently developed ONCV norm-conserving pseudopotentials ${ }^{34-36}$. The wave function energy cutoff was $80 \mathrm{Ry}$. We used a unit cell with formula $\mathrm{Li}_{8} \mathrm{Mn}_{16} \mathrm{O}_{32}$ and $4 \times 4 \times 4$ $\mathrm{k}$-point sampling. The cell was optimized at the PBE $+\mathrm{U}$ level of theory with a $\mathrm{U}_{\mathrm{Mn}}=3.5$. Previous studies showed that taking into account Jahn-Teller distortion and using hybrid or DFT $+\mathrm{U}$ levels of theory are essential for describing the charge localization that accompanies the two different oxidation states of $\mathrm{Mn}^{15,37}$. On average, the oxidation state of $\mathrm{Mn}$ is 3.5 , which turns to formally 3 and 4 , when the cubic unit cell is Jahn-Teller distorted into a tetragonal structure. It was also demonstrated that ferromagnetic (FM) and antiferromagnetic (AFM) arrangements of $\mathrm{Mn}$ ions are very close in energy, although the AFM ordering was shown to be slightly more stable ${ }^{15}$. More importantly, the optical properties were similar with AFM and FM ordering, we thus restricted our studies to FM ordering to avoid the complications arising from the multiple possible magnetic solutions when searching for the disproportionated state. To ensure the robustness of the results, we also performed PBE0 hybrid functional calculations. It was shown for many semiconductors that the mixing fraction entering the PBE0 hybrid functional should be chosen as the inverse of the high frequency dielectric constant. For LMO, the dielectric constant is 4.78 at the HSE06 level of theory ${ }^{15}$, suggesting that the original PBE0 mixing fraction of 0.25 might give rise to reliable quasiparticle band gaps. PBE0 calculations were carried out at the DFT $+U$ geometry with a

Monkhorst-Pack (MP) grid of $2 \times 2 \times 2$, a momentum transfer grid of $1 \times 1 \times 1$ and a reduced 80 Ry energy cutoff for the Fock operator. For the PBE0 calculations, we used a locally modified version of Quantum-Espresso to start PBE0 calculations with $\mathrm{PBE}+\mathrm{U}$ wave functions. This not only allowed us to reach convergence much faster than from a PBE starting point, but it also made sure that the right magnetic state is reached. We found that the energy difference between the ground state and the disproportionated state was $0.72 \mathrm{eV}$ at the PBE0 level of theory, which is in good qualitative agreement with that obtained with DFT $+\mathrm{U}(0.45 \mathrm{eV})$. See Supplementary Fig. 9 for a comparison of the DOS obtained at the DFT $+U$ and PBE0 levels of theory.
We monitored oxidation states and local magnetizations using atomic projected charges and spin densities in QE and then verified the results by using a Maximally Localized Wannier Function (MLWF) approach. In particular, we computed MLWFs in QBOX using the same pseudopotentials that we used in $\mathrm{QE}^{38}$. We used the PBE0 hybrid functional, a reduced MP grid of the Gamma-point only and a wave function energy cutoff of 60 Ry. Since LMO is a strongly ionic system, the Wannier centers are located in close proximity to atoms. We thus counted the number of Wannier centers close to each atom and defined the oxidation state as the number of valence electrons minus the number of Wannier centers associated with that atom. The magnetic moment was computed by subtracting the number of up spin Wannier centers from the number of down spin Wannier centers. This approach of assigning oxidation states to atoms in solids is similar to what has been proposed in the literature by Jang et al. ${ }^{39}$, where the oxidation state was defined using the number of Wannier centers that move with an atom if the atom is displaced by a lattice vector ${ }^{39,40}$.

As described in the main text, the ground state structure of bulk LMO contains alternating and ordered $\mathrm{Mn}^{3+}$ and $\mathrm{Mn}^{4+}$ sites. We first constrained an excess electron on several different $\mathrm{Mn}^{3+}$ sites (making the chosen site $\mathrm{Mn}^{2+}$ ) with the excess electron being spontaneously withdrawn from another $\mathrm{Mn}^{3+}$ site (to become $\mathrm{Mn}^{4+}$ ). Having optimized the structure under this charge constraint, we further re-optimized the structure with the constraint released. We obtained the disproportionated structure by constraining the charge at a Mn site. We tried several different methods to constrain an excess charge: (i) increasing/decreasing the $\mathrm{U}$ of the selected $\mathrm{Mn}$ atom, (ii) charging the supercell, (iii) constraining the $\mathrm{Mn}-\mathrm{O}$ bond lengths to $\sim 2.2 \AA$ (see Supplementary Fig. 10) for the Mn-O bond lengths in different Mn oxidation states). At the end, we found the disproportionated structure by a combination of (i) and (ii).

Although we performed our calculations on bulk LMO, we expect our results to qualitatively hold also for surfaces of LMO. To examine the effect of surfaces, we simulated a spherical stoichiometric cluster of LMO that has both high and low Miller index surfaces. The formula of the cluster was $\mathrm{Li}_{14} \mathrm{Mn}_{28} \mathrm{O}_{56}$ and the size was chosen so as to have bulk-like Mn atoms in the core. During the construction of the cluster we removed any Mn sites that had fewer than three Mn-O bonds. We found that under-coordinated $\mathrm{Mn}$ atoms ( $\mathrm{Mn}$ atoms that have only three $\mathrm{Mn}-\mathrm{O}$ bonds) naturally turn into $\mathrm{Mn}^{2+}$ without any energy barrier. Our result that disproportionation is more likely to happen on surfaces is further supported by the substantial experimental evidence that suggests that dissolution of $\mathrm{Mn}$ from the surface of LMO to the electrolyte is due to Mn disproportionation on the surface ${ }^{41}$ Furthermore, recent theoretical studies found that disproportionation of LMO might be an energetically favorable process on certain higher Miller index surfaces ${ }^{42}$.

\section{Data availability}

The data that support the findings of this study are available from the corresponding author upon request.

\section{Code availability}

The computer code that support the findings of this study are available from the corresponding author upon request.

Received: 30 June 2018; Accepted: 15 September 2019; Published online: 30 October 2019

\section{References}

1. Kang, K., Meng, Y. S., Bréger, J., Grey, C. P. \& Ceder, G. Electrodes with high power and high capacity for rechargeable lithium batteries. Science 311, 977-980 (2006).

2. Rolison, D. R. et al. Multifunctional 3D nanoarchitectures for energy storage and conversion. Chem. Soc. Rev. 38, 226-252 (2009).

3. Wang, Y. \& Cao, G. Developments in nanostructured cathode materials for high-performance lithium-ion batteries. Adv. Mater. 20, 2251-2269 (2008).

4. Bruce, P. G., Scrosati, B. \& Tarascon, J.-M. Nanomaterials for rechargeable lithium batteries. Angew. Chem. Int. Ed. 47, 2930-2946 (2008).

5. Goodenough, J. B. \& Park, K.-S. The Li-ion rechargeable battery: a perspective. J. Am. Chem. Soc. 135, 1167-1176 (2013).

6. Whittingham, M. S. Lithium batteries and cathode materials. Chem. Rev. 104, 4271-4302 (2004)

7. Ellis, B. L., Lee, K. T. \& Nazar, L. F. Positive electrode materials for Li-ion and Li-batteries. Chem. Mater. 22, 691-714 (2010).

8. Marom, R., Amalraj, S. F., Leifer, N., Jacob, D. \& Aurbach, D. A review of advanced and practical lithium battery materials. J. Mater. Chem. 21, 9938-9954 (2011).

9. Lu, J. et al. The role of nanotechnology in the development of battery materials for electric vehicles. Nat. Nanotechnol. 11, 1031-1038 (2016).

10. Thackeray, M. M., Johnson, P. J., de Picciotto, L. A., Bruce, P. G. \& Goodenough, J. B. Electrochemical extraction of lithium from $\mathrm{LiMn}_{2} \mathrm{O}_{4}$. Mater. Res. Bull. 19, 179-187 (1984). 
11. Hunter, J. C. Preparation of a new crystal form of manganese dioxide: $\lambda$ $\mathrm{MnO}_{2}$. J. Solid State Chem. 39, 142-147 (1981).

12. Ohzuku, T., Kitagawa, M. \& Hirai, T. Electrochemistry of manganese dioxide in lithium nonaqueous cell III. X-ray diffractional study on the reduction of spinel-related manganese dioxide. J. Electrochem. Soc. 137, 769-775 (1990).

13. Shiraishi, Y., Nakai, I., Tsubata, T., Himeda, T. \& Nishikawa, F. In situ transmission X-ray absorption fine structure analysis of the charge-discharge process in $\mathrm{LiMn}_{2} \mathrm{O}_{4}$, a rechargeable lithium battery material. J. Solid State Chem. 133, 587-590 (1997).

14. Kushida, K. \& Kuriyama, K. Observation of the crystal-field splitting related to the Mn-3d bands in spinel-LiMn2O4 films by optical absorption. Appl. Phys. Lett. 77, 4154-4156 (2000).

15. Hoang, K. Understanding the electronic and ionic conduction and lithium overstoichiometry in LiMn2O4 spinel. J. Mater. Chem. A 2, 18271-18280 (2014).

16. Mukerjee, S. et al. Structural evolution of LixMn2O4 in lithium-ion battery cells measured in situ using synchrotron X-ray diffraction techniques. $J$. Electrochem. Soc. 145, 466-472 (1998).

17. Würfel, P. \& Würfel, U. Physics of solar cells: from basic principles to advanced concepts. (Wiley-VCH, 2009).

18. Stoyanova, R., Gorova, M. \& Zhecheva, E. EPR of Mn4+ in spinels $\mathrm{Li}_{1+\mathrm{x}} \mathrm{Mn}_{2-\mathrm{x}} \mathrm{O}_{4}$ with $0 \leq \mathrm{x} \leq 0.1$. J. Phys. Chem. Solids 61, 609-614 (2000).

19. N. Zhecheva, E., Y. Gorova, M. \& K. Stoyanova, R. Microstructure of Li1 $+\mathrm{xMn}_{2-\mathrm{x}} \mathrm{O} 4$ spinels obtained from metal-organic precursors. J. Mater. Chem. 9, 1559-1567 (1999)

20. Abragam, A. \& Bleaney, B. Electron Paramagnetic Resonance of Transition Ions. 944 (Oxford University Press, 2012).

21. Pilbrow, J. R. Transition ion electron paramagnetic resonance. 738 (Clarendon Press, 1991).

22. Saponjic, Z. V. et al. Charge separation and surface reconstruction: $\mathrm{A} \mathrm{Mn}^{2+}$ doping study. J. Phys. Chem. B 110, 25441-25450 (2006).

23. Misra, S. K. Interpretation of Mn2 + EPR spectra in disordered materials. Appl. Magn. Reson. 10, 193-216 (1996).

24. Kanamura, K., Naito, H., Yao, T. \& Takehara, Z.-i Structural change of the LiMn2O4 spinel structure induced by extraction of lithium. J. Mater. Chem. 6 , 33-36 (1996)

25. Ramana, C. V., Massot, M. \& Julien, C. M. XPS and Raman spectroscopic characterization of LiMn2O4 spinels. Surf. Interface Anal. 37, 412-416 (2005).

26. Rodríguez-Carvajal, J., Rousse, G., Masquelier, C. \& Hervieu, M. Electronic crystallization in a lithium battery material: columnar ordering of electrons and holes in the spinel LiMn2O4. Phys. Rev. Lett. 81, 4660-4663 (1998).

27. Hodes, G., Manassen, J. \& Cahen, D. Photoelectrochemical energy conversion and storage using polycrystalline chalcogenide electrodes. Nature 261, 403-404 (1976).

28. Li, N., Wang, Y., Tang, D. \& Zhou, H. Integrating a Photocatalyst into a Hybrid Lithium-Sulfur Battery for Direct Storage of Solar Energy. Angew. Chem. 127, 9403-9406 (2015).

29. Paolella, A. et al. Light-assisted delithiation of lithium iron phosphate nanocrystals towards photo-rechargeable lithium ion batteries. Nat. Commun. 8, 14643 (2017).

30. Ammundsen, B., Burns, G. R., Islam, M. S., Kanoh, H. \& Rozière, J. Lattice dynamics and vibrational spectra of lithium manganese oxides: a computer simulation and spectroscopic study. J. Phys. Chem. B 103, 5175-5180 (1999).

31. Chitra, S. et al. Characterization and electrochemical studies of LiMn2O4 cathode materials prepared by combustion method. J. Electroceram. 3, 433-441 (1999).

32. Hwang, S.-J., Park, D.-H., Choy, J.-H. \& Campet, G. Effect of chromium substitution on the lattice vibration of spinel lithium manganate: A new interpretation of the raman spectrum of LiMn2O4. J. Phys. Chem. B 108 , 12713-12717 (2004).

33. Paolo, G. et al. QUANTUM ESPRESSO: a modular and open-source software project for quantum simulations of materials. J. Phys.: Condens. Matter 21, 395502 (2009).

34. Hamann, D. R. Optimized norm-conserving Vanderbilt pseudopotentials. Phys. Rev. B 88, 085117 (2013).

35. Schlipf, M. \& Gygi, F. Optimization algorithm for the generation of ONCV pseudopotentials. Comput. Phys. Commun. 196, 36-44 (2015).

36. Lejaeghere, $\mathrm{K}$. et al. Reproducibility in density functional theory calculations of solids. Science 351, aad3000 (2016).

37. Warburton, R. E., Iddir, H., Curtiss, L. A. \& Greeley, J. Thermodynamic stability of low- and high-index spinel LiMn2O4 surface terminations. ACS Appl. Mater. Interfaces 8, 11108-11121 (2016).

38. Gygi, F. Architecture of Qbox: a scalable first-principles molecular dynamics code. IBM J. Res. Dev. 52, 137-144 (2008).

39. Jiang, L., Levchenko, S. V. \& Rappe, A. M. Rigorous definition of oxidation states of ions in solids. Phys. Rev. Lett. 108, 166403 (2012).
40. Chen, J., Wu, X. \& Selloni, A. Electronic structure and bonding properties of cobalt oxide in the spinel structure. Phys. Rev. B 83, 245204 (2011).

41. Amos, C. D., Roldan, M. A., Varela, M., Goodenough, J. B. \& Ferreira, P. J. Revealing the reconstructed surface of $\mathrm{Li}\left[\mathrm{Mn}_{2}\right] \mathrm{O}_{4}$. Nano Lett. 16, 2899-2906 (2016).

42. Scivetti, I. \& Teobaldi, G. (Sub)surface-promoted disproportionation and absolute band alignment in high-pwer LiMn2O4 cathodes. J. Phys. Chem. C 119, 21358-21368 (2015)

\section{Acknowledgements}

This work was supported as part of the Center for Electrochemical Energy Science (CEES), an Energy Frontier Research Center funded by the U.S. Department of Energy, Office of Science, Basic Energy Sciences. A.L. and C.J. thank J. Blauwkamp and M. Piernas for technical assistance with cell assembly, T. Rajh and E. Rozhkova for lending the Xe lamp and associated power supply, and L. Utschig and A. Ahmed for helpful discussions. We thank Aude Annick Heidet for technical assistance with sputtering. The work of M.V. was supported by the Laboratory Directed Research and Development (LDRD) funding from Argonne National Laboratory, provided by the Director, Office of Science, of the U.S. DOE under Contract No. DE-AC02-06CH11357. We gratefully acknowledge the computing resources provided on Blues, a high-performance computing cluster operated by the Laboratory Computing Resource Center at Argonne National Laboratory. Further resources were provided by the National Energy Research Scientific Computing Center, a DOE Office of Science User Facility supported by the Office of Science of the U.S. Department of Energy under Contract No. DE-AC02-05CH11231. This work was performed, in part, at the Center for Nanoscale Materials, a U.S. Department of Energy Office of Science User Facility under Contract No. DE-AC02$06 \mathrm{CH} 11357$ (R.S. and V.M.). This material is based upon work supported by the U.S. Department of Energy, Office of Science, Office of Basic Energy Sciences, Division of Chemical Sciences, Geosciences, and Biosciences, under contract number DE-AC0206CH11357 at Argonne National Laboratory (J.N. and O.G.P.)

\section{Author contributions}

A.L. and C.J. conceived, developed, designed, and carried out the experiments; M.V. carried out density functional theory modeling and M.V., H.I., and L.A.C. were responsible for the theoretical computations. J.N. and O.P. performed electron paramagnetic resonance measurements; R.D.S. performed transient absorption spectroscopy; W.M.D. carried out additional electrochemical experiments; V.A.M. performed Raman spectroscopy; E.L. contributed the design of electrodes; B.I. fabricated the thin film. C.J. supervised the project and all authors contributed to development of the manuscript and to discussions as the project developed. All authors discussed the results and reviewed the manuscript.

\section{Competing interests}

The authors declare no competing interests.

\section{Additional information}

Supplementary information is available for this paper at https://doi.org/10.1038/s41467019-12863-6.

Correspondence and requests for materials should be addressed to C.S.J.

Peer review information Nature Communications thanks the anonymous reviewer(s) for their contribution to the peer review of this work.

Reprints and permission information is available at http://www.nature.com/reprints

Publisher's note Springer Nature remains neutral with regard to jurisdictional claims in published maps and institutional affiliations.

Open Access This article is licensed under a Creative Commons Attribution 4.0 International License, which permits use, sharing, adaptation, distribution and reproduction in any medium or format, as long as you give appropriate credit to the original author(s) and the source, provide a link to the Creative Commons license, and indicate if changes were made. The images or other third party material in this article are included in the article's Creative Commons license, unless indicated otherwise in a credit line to the material. If material is not included in the article's Creative Commons license and your intended use is not permitted by statutory regulation or exceeds the permitted use, you will need to obtain permission directly from the copyright holder. To view a copy of this license, visit http://creativecommons.org/ licenses/by/4.0/.

This is a U.S. Government work and not under copyright protection in the US; foreign copyright protection may apply 2019 